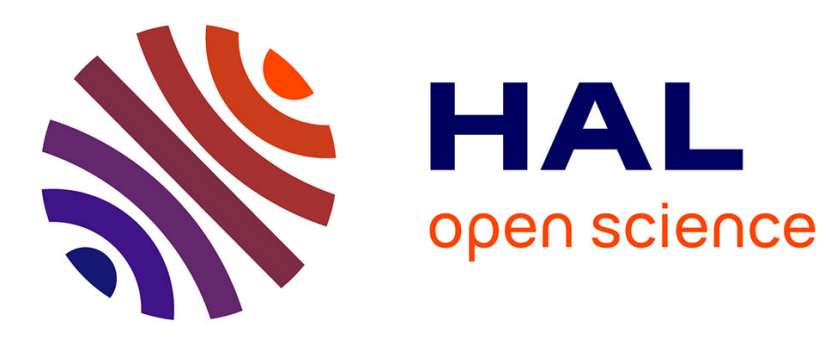

\title{
Travail et tentatives de suicide
}

Stéphane François, Marie-Pierre Guiho-Bailly, Bénédicte Gohier, Jean-Bernard Garré, Julie Bodin, Aurélie François, Yves Roquelaure

\section{To cite this version:}

Stéphane François, Marie-Pierre Guiho-Bailly, Bénédicte Gohier, Jean-Bernard Garré, Julie Bodin, et al. Travail et tentatives de suicide. Archives des Maladies Professionnelles et de L'Environnement, 2012, 73 (2), pp.112 - 9. 10.1016/j.admp.2012.03.001 . hal-03389588

\section{HAL Id: hal-03389588 \\ https://univ-angers.hal.science/hal-03389588}

Submitted on 21 Oct 2021

HAL is a multi-disciplinary open access archive for the deposit and dissemination of scientific research documents, whether they are published or not. The documents may come from teaching and research institutions in France or abroad, or from public or private research centers.
L'archive ouverte pluridisciplinaire HAL, est destinée au dépôt et à la diffusion de documents scientifiques de niveau recherche, publiés ou non, émanant des établissements d'enseignement et de recherche français ou étrangers, des laboratoires publics ou privés. 


\title{
Travail et tentatives de suicide
}

\author{
Work and suicide attempts \\ S. François ${ }^{a, *}$, M.-P. Guiho-Bailly ${ }^{a}$, B. Gohier ${ }^{b}$, J.-B. Garre ${ }^{b}$, J. Bodin $^{a}$, A. François ${ }^{a}$, \\ Y. Roquelaure ${ }^{a}$ \\ a Laboratoire d'ergonomie et d'épidémiologie en santé au travail, faculté de médecine \\ d'Angers, 1, rue Haute-de-Reculée, 49045 Angers cedex, France \\ ${ }^{\mathrm{b}}$ Département de psychiatrie et de psychologie médicale, CHU d'Angers, 4, rue Larrey, \\ $\checkmark 49933$ Angers cedex 9, France
} SciVerse ScienceDirect www.sciencedirect.com

\section{Summary}

Purpose of the study. A large increase in work-related suicides has been noted in the recent years. This applies to all company staff, from agent to manager. To consider a prevention of work-related suicides, it is important to define them in terms of quantity and quality. To partially address this problem, this study aims at clarifying the professional characteristics of a sample of suicide attempters. Method. This 6-months study included the suicide attempters in employment, aged 18-65 years, hospitalized at the CHU of Angers. 87 consecutive suicide attempters have responded to questionnaires that focused on job characteristics.

Results. Suicide attempters in the group "work-related suicide attempts (SA)" rather describe the presence of pathogenic element of work organizations including an overload of work, deadlines and frequent disturbances. In addition, it also reports more conflicts in the company. This concerns mainly managers and intermediate professions. The proportion of men is higher among the suicide attempters establishing a link between SA and their work, but women are also affected.

Discussion. The link between work and SA seems to have more to do with work organization, relations with superiors and colleagues, poor recognition at work than exposure to physical constraints, working hours and type of employment contract.

Conclusion. Prevention of work-related suicide must go through a reflection on the organization of work, social relations within the company and promoting the recognition of real work.

(C) 2012 Published by Elsevier Masson SAS.

Keywords: Suicide attempt, Suicide, Organization, Work, Psychosocial factors

\section{Résumé}

Objectif. L'irruption médiatique de suicides en lien avec le travail constitue un élément marquant de ces dernières années. Ce phénomène doit mobiliser tous les acteurs de l'entreprise, de l'agent au directeur. Pour envisager une prévention des suicides liés au travail, il est important de les définir en termes quantitatif et qualitatif. Afin de répondre en partie à cette problématique, cette étude vise à préciser les caractéristiques professionnelles d'un échantillon de suicidants dont une partie annonce un lien entre leur passage à l'acte suicidaire et leur travail.

Méthodes. Cette étude d'une durée de six mois a inclus les suicidants actifs ayant un emploi, âgés de 18 à 65 ans, hospitalisés au CHU d'Angers. Au total, 87 suicidants consécutifs ont répondu aux questionnaires portant sur les caractéristiques du travail.

Résultats. Les suicidants appartenant au groupe « tentatives de suicide (TS) liées au travail » décrivent davantage la présence d'éléments d'organisations pathogènes du travail comprenant une surcharge de travail, des délais à respecter et des dérangements fréquents. De plus, ils rapportent également davantage de conflits dans l'entreprise. Cela concerne en majorité les cadres et les professions intermédiaires. La proportion des hommes est plus importante parmi les suicidants établissant un lien entre leur TS et leur travail, mais les femmes sont aussi concernées.

Discussion. Le lien entre le travail et les TS semble davantage provenir de l'organisation du travail, des rapports avec la hiérarchie et les collègues, ainsi que d'une mauvaise reconnaissance au travail, que de l'exposition aux contraintes physiques, aux horaires de travail et au type de contrat de travail.

Conclusion. La prévention du suicide lié au travail doit passer par une réflexion sur l'organisation du travail, les rapports sociaux

\footnotetext{
* Auteur correspondant.

e-mail : st.francois@hotmail.fr

$1775-8785 \mathrm{X} / \$$ - see front matter $\odot 2012$ Publié par Elsevier Masson SAS

10.1016/j.admp.2012.03.001 Archives des Maladies Professionnelles et de l'Environnement 2012;73:112-119

112
} 
au sein de l'entreprise et la promotion de la reconnaissance du travail réel.

(C) 2012 Publié par Elsevier Masson SAS.

Mots clés : Tentatives de suicide, Suicide, Organisation du travail, Travail, Facteur psychosocial

\section{Introduction}

Selon les sociologues, le suicide donne un éclairage particulier sur notre société [1]. On constate depuis quelques années une augmentation des phénomènes suicidaires présentant un lien possible avec le travail, que ce soit par leurs modes ou leurs lieux de réalisation, ou par la présence de traces écrites dénonçant le travail. Ce phénomène préoccupant, reflet de dysfonctionnements graves dans le monde du travail actuel, est présent dans tous les secteurs d'activités. Les nouvelles organisations du travail tendent à isoler les salariés et à les mettre en concurrence. C'est alors seul qu'ils font face aux agressions qu'ils reçoivent au travail, à l'intensification croissante des rythmes de travail et à la réduction des marges de manœuvres [2]. C'est dans ce contexte qu'a été rédigé l'accord national interprofessionnel sur le stress au travail signé le o2 juillet 2008.

Malgré ce constat, le lien entre le travail et les phénomènes suicidaires est souvent difficile à établir s'il est réalisé en dehors du travail et sans trace écrite incriminant ce dernier, ce qui entraîne une connaissance partielle du problème et donc une mauvaise visibilité des facteurs de pénibilité mentale liés au travail qui en seraient responsables [3].

Dans le but d'affiner nos connaissances dans ce domaine, nous avons analysé les conditions de travail d'un échantillon de suicidant qui travaillaient au moment de la tentative de suicide (TS) et dont une partie indiquait l'existence d'un lien entre leur passage à l'acte suicidaire et leur travail.

\section{Matériel et méthode}

Cette étude pilote a été réalisée par le laboratoire d'ergonomie et d'épidémiologie en santé au travail en collaboration avec le département de psychiatrie et de psychologie médicale du CHU Angers. Elle a fait l'objet d'un accord CNIL.

\section{Population d'étude}

Dans cette enquête descriptive étaient inclus tous les suicidant âgés de 18 à 65 ans, hospitalisés du 2 novembre 2008 au 15 mai 2009 à l'unité médico-psychosociale du CHU Angers. Il s'agit d'une unité spécialisée dans la prise en charge de la crise suicidaire de patients adultes admis initialement en réanimation (74 \%) ou aux urgences médicales adultes (26\%) et consentant aux soins. Les suicidants exclus de l'étude étaient ceux ayant fugué ou quitté le service de soin contre avis médical et n'ayant pas rendu le questionnaire, ceux présentant des troubles de conscience et ceux ayant déjà été inclus dans l'étude lors d'une TS antérieure. Au total, 150 suicidants ont été hospitalisés au CHU d'Angers suite à une TS et 144 suicidants ont accepté de participer à l'étude. Parmi eux, 87 étaient actifs avec un emploi et constituent l'échantillon étudié.

\section{Recueil des données}

Un enquêteur a proposé un auto-questionnaire à chaque suicidant actif ayant un emploi. Ce questionnaire avait pour objectif de renseigner la sphère professionnelle du patient. II est basé sur des questionnaires existants, utilisés en santé au travail (Karasek, Samotrace et Siegrist) et un questionnaire intitulé " Travail et santé mentale » créé par les investigateurs de l'étude.

Le questionnaire de Karasek, en examinant la latitude décisionnelle (LD), la demande psychologique (DP) et le soutien social au travail permet de situer le salarié dans une des quatre catégories suivantes : tendue (LD faible et DP forte), détendue (LD forte et DP faible), active (LD forte et DP forte) ou passive (LD faible et DP faible) [4,5]. La catégorie tendue est également appelée job strain. Cette dernière associée à un faible soutien professionnel correspond à la situation de travail la plus péjorative appelée iso-strain. Les scores ont été dichotomisés à partir des médianes nationales issues de l'enquête Sumer 2003 (70 pour la latitude décisionnelle, 21 pour la charge mentale et 23 pour le soutien social).

Le questionnaire Samotrace est un système national de surveillance épidémiologique de la santé mentale en lien avec le travail $[6,7]$. Son volet épidémiologie en entreprise est utilisé ici afin d'analyser les caractéristiques de l'organisation et des conditions de travail (catégorie socioprofessionnelle, contrats de travail, horaires, contraintes...).

Le questionnaire de Siegrist recherche la présence d'un déséquilibre dans le rapport investissement récompense au travail, qui favorise l'apparition de pathologies [8,9]. L'utilisation de ce questionnaire dans son intégralité nous a semblé trop lourde pour être proposé à des suicidants et nous avons préféré n'utiliser que sept questions parmi les 72 qui composent ce questionnaire dans sa version longue. Les questions choisies ont permis d'explorer le niveau d'équilibre existant entre les investissements au travail et les rétributions subjectives et matérielles obtenues en retour. 
Le questionnaire " Travail et santé mentale " recherche la présence d'éléments d'organisations pathogènes du travail décrits par la littérature, que le patient aurait rencontré dans les 12 derniers mois [5,10-15].

Il était demandé aux patients d'apprécier le lien existant entre leurs passages à l'acte suicidaire et leur travail. Au total, quatre niveaux de lien leur étaient proposés (lien direct essentiel, lien direct non essentiel, lien douteux et lien nul). En raison du faible effectif de l'échantillon, deux groupes ont été créés : "TS liées au travail " qui regroupe les modalités " lien direct essentiel " et " lien direct non essentiel " et " TS non liées au travail " qui regroupe les modalités "lien douteux " et "lien nul ".

Un délai de 48 heures entre la TS et la remise des autoquestionnaires était respecté afin d'éviter que les effets toxiques secondaire aux intoxications médicamenteuses volontaires induisent des réponses peu fiables.

\section{Analyses statistiques}

La distribution de l'échantillon en fonction du genre et de l'âge a été comparée à celle des Pays de la Loire et de la France [16]. Pour les données qualitatives, un test de $\mathrm{Chi}_{2}$ a été utilisé et un test de comparaison de moyennes a permis de comparer l'âge. Le seuil de signification a été fixé à o,05 et tous les tests étaient bilatéraux.

Ensuite, une analyse descriptive des conditions de travail des groupes " TS liées au travail » et " TS non liées au travail " a été réalisée.
L'ensemble des analyses statistiques ont été réalisées avec le logiciel SPSS version 17 .

\section{Résultats}

Les questionnaires remplis présentaient peu de données manquantes (de o à 9,2 \% selon les questions).

\section{Analyse des questionnaires}

Parmi les 87 suicidants actifs au moment de leur TS, $32 \%$ (soit 28 suicidants) estiment que leur TS est liée au travail. Cela concerne $40 \%$ des hommes et $28 \%$ des femmes.

Toutes les catégories socioprofessionnelles sont touchées, mais les employés et les professions intermédiaires sont les plus représentés dans le groupe "TS liées au travail ". À noter également que cinq des neuf cadres et professions intellectuelles supérieures établissent un lien entre leur TS et leur travail. Dans la majorité des cas, les suicidants du groupe « TS liées au travail " sont en CDI et ne présentent pas davantage de contrats professionnels précaires que les suicidants du groupe "TS non liées au travail ".

Les suicidants du groupe "TS liées au travail " sont âgés en moyenne de 34 ans alors que les suicidants du groupe «TS non liées au travail " sont âgés en moyenne de 36 ans (tableau I). Les horaires de travail sont assez similaires dans les deux groupes. Le travail à temps plein est majoritairement rencontré ( $\geq 60 \%)$. Se coucher après minuit ou se lever avant cing heures à cause du travail est peu fréquent. Travailler la nuit

Tableau I

Caractéristiques sociodémographiques et professionnelles des groupes " TS liées au travail " et " TS non liées au travail ".

\begin{tabular}{|c|c|c|c|c|c|c|}
\hline & \multicolumn{2}{|c|}{$\begin{array}{l}\text { TS liées au } \\
\text { travail }(n=28)\end{array}$} & \multicolumn{2}{|c|}{$\begin{array}{l}\text { TS non liées au } \\
\text { travail }(n=59)\end{array}$} & \multicolumn{2}{|c|}{ Total $(n=87)$} \\
\hline & $n$ & $\%$ & $n$ & $\%$ & $n$ & $\%$ \\
\hline \multicolumn{7}{|l|}{ Sexe } \\
\hline Femme & 16 & 57 & 41 & 70 & 57 & 66 \\
\hline Homme & 12 & 43 & 18 & 30 & 30 & 34 \\
\hline \multicolumn{7}{|l|}{ Contrat de travail } \\
\hline CDI & 23 & 82 & 37 & 68 & 60 & 73 \\
\hline CDD & 2 & 7 & 14 & 26 & 16 & 20 \\
\hline Apprentis & 1 & 4 & 0 & 0 & 1 & 1 \\
\hline Intérimaires & 0 & 0 & o & o & 0 & o \\
\hline Autres & 2 & 7 & 3 & 6 & 5 & 6 \\
\hline \multicolumn{7}{|l|}{ Catégories socioprofessionnelles } \\
\hline Employés & 10 & 36 & 14 & 26 & 24 & 29 \\
\hline Ouvriers & 4 & 14 & 19 & 35 & 23 & 28 \\
\hline Professions intermédiaires & 7 & 25 & 14 & 26 & 21 & 25 \\
\hline Cadres, PIS $^{\mathrm{a}}$ & 5 & 18 & 4 & 7 & 9 & 11 \\
\hline Artisans, commerçants et chefs d'entreprise & 1 & 4 & 3 & 6 & 4 & 5 \\
\hline Agriculteur exploitant & 1 & 4 & 1 & 2 & 2 & 2 \\
\hline Âge moyen en années & 34 & & 36 & & 35 & \\
\hline
\end{tabular}

${ }^{a}$ Professions intellectuelles supérieures. 
est encore plus rare. Les suicidants n'évoquant pas de lien entre leur TS et leur travail sont plus nombreux à travailler plus d'un samedi sur deux dans l'année (40\% versus $18 \%$ ). En revanche, les suicidants du groupe TS liées au travail sont davantage à travailler plus de 10 heures par jour (36\% versus $21 \%$ ) et à avoir un temps de trajet domicile-travail aller et retour supérieur ou égal à une heure (22\% versus $13 \%$ ). Globalement, les deux groupes estiment en majorité que leurs horaires de travail s'accordent bien avec leurs engagements sociaux et familiaux (tableau II).

L'exposition aux contraintes physiques est comparable dans les deux groupes. Ils évoquent en premier lieu les postures pénibles et les ports de charges lourdes (tableau II).
Les facteurs d'organisations pathogènes du travail sont également plus fréquemment retrouvés dans le groupe des TS liées au travail. Les suicidants de ce groupe évoquent davantage des délais à respecter, des dérangements fréquents, une surcharge de travail, des exigences du public à supporter, des tâches mal définies, des injonctions contradictoires, une qualité et une rentabilité difficile à atteindre, un travail en sous effectif, des mutations subies, des glissements de tâches, une sous-charge de travail et une rémunération au rendements (tableau II).

Les suicidants du groupe TS liées au travail évoquent aussi davantage de mauvaises relations sociales en entreprise. Ils se plaignent en premier lieu de stress intense, de tension avec la

Tableau II

Contraintes physiques et organisationnelles décrites par les suicidants.

\begin{tabular}{|c|c|c|c|c|c|c|}
\hline & \multicolumn{2}{|c|}{$\begin{array}{l}\text { TS liées au travail } \\
(n=28)\end{array}$} & \multicolumn{2}{|c|}{$\begin{array}{l}\text { TS non liées au } \\
\text { travail }(n=59)\end{array}$} & \multicolumn{2}{|c|}{ Total $(n=87)$} \\
\hline & $n$ & $\%$ & $n$ & $\%$ & $n$ & $\%$ \\
\hline \multicolumn{7}{|l|}{ Temps de travail } \\
\hline $20 \mathrm{~h}$ à $34 \mathrm{~h}$ & 4 & 14 & 13 & 24 & 17 & 21 \\
\hline $35 \mathrm{~h}$ à $46 \mathrm{~h}$ & 18 & 64 & 33 & 60 & 51 & 61 \\
\hline Autres & 6 & 22 & 9 & 16 & 15 & 18 \\
\hline $\begin{array}{l}\text { Horaires de travail s'accordant bien avec les } \\
\text { engagements sociaux et familiaux }\end{array}$ & 17 & 61 & 39 & 71 & 56 & 67 \\
\hline Travailler plus d'1 samedi/2 & 5 & 18 & 23 & 40 & 28 & 33 \\
\hline Travailler plus de $10 \mathrm{~h}$ par jour & 10 & 36 & 12 & 21 & 22 & 26 \\
\hline Lever avant $5 \mathrm{~h}$ pour le travail & 5 & 18 & 9 & 16 & 14 & 16 \\
\hline Travailler plus d'1 dimanche/2 & 4 & 14 & 10 & 17 & 14 & 16 \\
\hline Trajet domicile-travail $>1 \mathrm{~h}$ aller et retour & 6 & 22 & 7 & 13 & 13 & 16 \\
\hline Coucher après minuit pour le travail & 3 & 11 & 9 & 16 & 12 & 14 \\
\hline Travailler la nuit & 1 & 4 & 5 & 9 & 6 & 7 \\
\hline \multicolumn{7}{|l|}{ Contraintes physiques } \\
\hline Postures pénibles & 13 & 48 & 28 & 48 & 41 & 48 \\
\hline Ports de charges lourdes & 13 & 46 & 25 & 43 & 38 & 44 \\
\hline Exposition aux poussières ou aux fumées & 7 & 25 & 17 & 29 & 24 & 28 \\
\hline Travail répétitif & 7 & 25 & 13 & 23 & 20 & 24 \\
\hline Exposition aux produits chimiques & 7 & 25 & 12 & 21 & 19 & 22 \\
\hline Bruits intenses & 7 & 25 & 11 & 19 & 18 & 21 \\
\hline Travail posté & 4 & 14 & 8 & 14 & 12 & 14 \\
\hline Exposition aux intempéries & 4 & 14 & 7 & 12 & 11 & 13 \\
\hline \multicolumn{7}{|l|}{ Éléments d'organisations pathogènes du travail } \\
\hline Rotations & 13 & 46 & 30 & 54 & 43 & 51 \\
\hline Surcharge de travail & 18 & 64 & 22 & 40 & 40 & 48 \\
\hline Délai à respecter & 21 & 75 & 17 & 31 & 38 & 46 \\
\hline Souvent dérangé & 19 & 68 & 18 & 33 & 37 & 45 \\
\hline Injonctions contradictoires & 15 & 54 & 17 & 31 & 32 & 39 \\
\hline Travail en sous effectif & 14 & 50 & 18 & 33 & 32 & 39 \\
\hline Manque de moyens & 11 & 39 & 17 & 31 & 28 & 34 \\
\hline Rentabilité difficile à atteindre & 15 & 54 & 13 & 24 & 28 & 34 \\
\hline Qualité difficile à atteindre & 15 & 54 & 11 & 20 & 26 & 32 \\
\hline Tâches mal définies & 17 & 61 & 8 & 15 & 25 & 30 \\
\hline Système d'évaluation non professionnelle & 10 & 37 & 14 & 26 & 24 & 30 \\
\hline Glissements de tâches & 7 & 26 & 6 & 11 & 13 & 16 \\
\hline Mutation subie & 8 & 29 & 3 & 6 & 11 & 13 \\
\hline Rémunération aux rendements & 7 & 25 & 4 & 7 & 11 & 13 \\
\hline Sous-charge de travail & 4 & 14 & 1 & 2 & 5 & 6 \\
\hline
\end{tabular}


Tableau III

Ambiances au travail décrites par les suicidants.

\begin{tabular}{|c|c|c|c|c|c|c|}
\hline & \multicolumn{2}{|c|}{$\begin{array}{l}\text { TS liées au } \\
\text { travail }(n=28)\end{array}$} & \multicolumn{2}{|c|}{$\begin{array}{l}\text { TS non liées au } \\
\text { travail }(n=59)\end{array}$} & \multicolumn{2}{|c|}{ Total $(n=87)$} \\
\hline & $n$ & $\%$ & $n$ & $\%$ & $n$ & $\%$ \\
\hline \multicolumn{7}{|l|}{ Vécu, ambiance et relations au travail } \\
\hline Supporter les exigences du public & 18 & 64 & 31 & 56 & 49 & 59 \\
\hline Stress intense & 24 & 86 & 21 & 39 & 45 & 54 \\
\hline Tension avec la hiérarchie & 18 & 64 & 11 & 20 & 29 & 35 \\
\hline Menaces ou humiliations & 12 & 43 & 11 & 20 & 23 & 28 \\
\hline Conscience professionnelle heurtée & 16 & 57 & 6 & 11 & 22 & 27 \\
\hline Tension avec les collègues & 10 & 36 & 9 & 16 & 19 & 23 \\
\hline Avertissement & 7 & 26 & 6 & 11 & 13 & 16 \\
\hline Menace de licenciement & 7 & 26 & 4 & 7 & 11 & 13 \\
\hline Discriminations liées à l'âge & 3 & 11 & 5 & 9 & 8 & 10 \\
\hline Violences physiques & 0 & o & 5 & 9 & 5 & 6 \\
\hline Discriminations liées au sexe & 3 & 11 & 1 & 2 & 4 & 5 \\
\hline Discriminations liées au handicap & 4 & 14 & 0 & $\mathrm{O}$ & 4 & 5 \\
\hline \multicolumn{7}{|l|}{ Reconnaissance au travail } \\
\hline Salaires insatisfaisants & 18 & 64 & 25 & 46 & 43 & 52 \\
\hline $\begin{array}{l}\text { Promotions insatisfaisantes } \\
\text { Je ne reçois pas le respect que je mérite }\end{array}$ & 14 & 50 & 15 & 29 & 29 & 36 \\
\hline de mes supérieurs & 15 & 54 & 13 & 24 & 28 & 34 \\
\hline Formation initiale non concordant au poste & 8 & 29 & 17 & 31 & 25 & 30 \\
\hline $\begin{array}{l}\text { Traitement injuste au travail } \\
\text { Je ne reçois pas le respect que je mérite }\end{array}$ & 13 & 46 & 7 & 13 & 20 & 24 \\
\hline de mes collègues & 8 & 29 & 7 & 13 & 15 & 18 \\
\hline
\end{tabular}

hiérarchie. Ils évoquent ensuite subir des menaces ou des humiliations et des tensions avec les collègues.

Leur conscience professionnelle est davantage heurtée que celle des suicidants du groupe "TS non liées au travail " (tableau III).

Le manque de reconnaissance professionnelle passant par les rétributions symboliques est plus souvent mentionné par les suicidants posant le lien entre leur passage à l'acte et leur travail. Ils estiment ne pas recevoir le respect qu'ils méritent de la part de leurs supérieurs et être traités injustement à leur travail (tableau III).

Les suicidants appartenant au groupe "TS liées au travail " sont davantage en situation tendue et active au travail que les

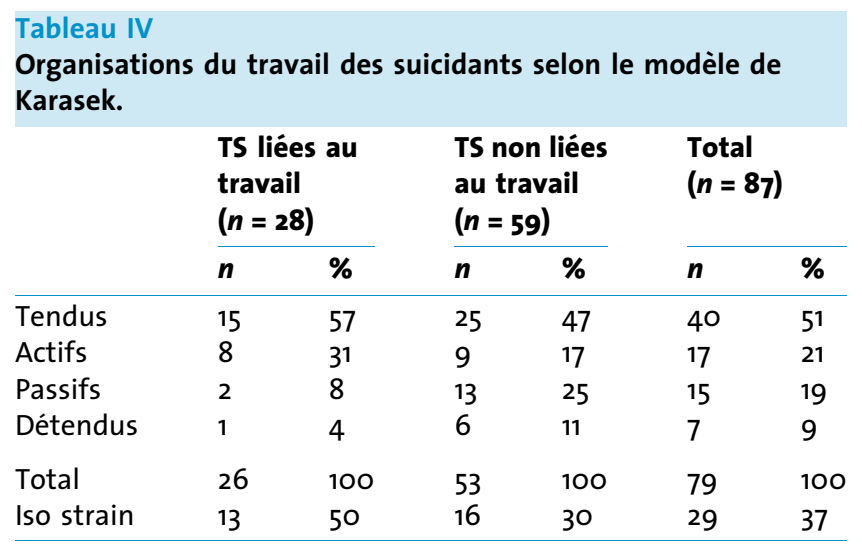

suicidants appartenant au groupe "TS non liées au travail ". Ils sont également davantage en situation d'iso strain (tableau IV).

\section{Discussion}

\section{Intérêts et limites de l'étude}

Cette étude a été réalisée à l'unité médico-psycho-sociale du CHU d'Angers, principal service d'accueil en urgence des crises suicidaires dans le Maine et Loire, ce qui permet un bon recrutement des suicidants du département, bien que certains ne soient pas admis dans ce service (admission dans un autre service et TS non hospitalisées). La présente étude apporte un éclairage particulier sur les conditions de travail décrites par les suicidants de l'échantillon qui annonçaient un lien direct entre leur travail et leur geste suicidaire. Elle a également permis une description détaillée des caractéristiques professionnelles d'une population de suicidants [16]. L'appréhension de ces notions est toujours délicate.

Il est important de souligner le fait que le lien entre la tentative de suicide et le travail repose sur un sentiment subjectif du patient et ne fait pas intervenir d'expertise médicale. De plus, l'échantillon étudié est de petite taille. L'ensemble de ces éléments implique de réaliser une interprétation prudente des résultats. 
Comparaison des caractéristiques " genre et âge " de l'échantillon à celles des populations de la France et des Pays de la Loire

On constate dans notre échantillon, une moyenne d'âge plus jeune et une proportion de femmes supérieure à celle des populations de référence [16]. Cela correspond aux descriptions faite dans la littérature concernant les populations de suicidants $[17,18]$.

\section{Une moyenne d'âge proche entre les deux groupes}

L'impact des conditions de travail sur la santé des travailleurs en fonction de leurs âges a été bien documenté [19]. Le travailleur vieillissant présente souvent une fragilité physiologique plus importante, mais il a acquis des savoirs-faire de métier, tiré de son expérience, lui permettant d'être performant. Lorsque les marges de manœuvres sont trop réduites, l'équilibre entre les capacités de l'individu et les exigences de la tâche, nécessaire à la préservation de la santé peut ne plus être suffisant.

Cependant, notre étude, du fait des petits effectifs concernés, ne permet pas de conclure à une différence concernant l'âge entre les groupes "TS liées au travail et " TS non liées au travail ». Au contraire, la moyenne d'âge des deux groupes est proche (tableau I). L'influence de l'âge dans les processus suicidaires liés au travail nécessite d'autres travaux.

\section{Pas de différence en ce qui concerne les catégories socioprofessionnelles, l'exposition aux contraintes physiques, le type de contrat et les horaires de travail}

Les différences existantes en ce qui concerne les conditions de travail des différentes catégories socioprofessionnelles soit décrites dans la littérature [20,21]. Cependant, ces dernières semblent toutes touchées par les tentatives de suicide en lien avec le travail. Cela est confirmé par le fait que les deux groupes de l'étude sont exposés de la même façon aux contraintes physiques (bruits, poussières, ports de charges lourdes,...). Le lien existant entre les TS et le travail pourrait ne pas se situer au niveau des contraintes physiques. Il en est de même en ce qui concerne le type de contrat et les horaires de travail des suicidants. II n'a pas était retrouvé plus de contrat de travail précaires ni davantage de temps partiel parmi les suicidants annonçant une relation étroite entre leur passage à l'acte et leur travail.

\section{Une proportion d'homme supérieure dans le groupe TS liées au travail}

La littérature décrit bien sous le terme de division sexuelle du travail, la différence existante entre les conditions de travail des hommes et des femmes. Ces dernières sont souvent mal reconnues à la vue de la qualification des postes qu'elles occupent. Elles réalisent souvent des travaux exigeant un certain niveau de finesse et sont davantage exposées aux exigences du public. Les hommes quant à eux sont davantage exposés aux contraintes physiques importantes (ports de charges lourdes, gestes répétitifs, maintien de postures contraignantes,...). Au-delà des contraintes physiques liées au travail, le rapport subjectif au travail peut-être différent selon le sexe. Dans cette étude, on retrouve une proportion d'hommes plus importante dans le groupe des TS liées au travail, mais on constate que les deux sexes sont touchés par ce phénomène.

\section{Davantage d'organisations pathogènes du travail décrites par les suicidants établissant un lien entre leur travail et leur passage à l'acte suicidaire}

Certains éléments d'organisations du travail connues pour leur caractère pathogène $[10,13,22]$ sont davantage décrits par les suicidants évoquant un lien entre leur travail et leur TS. Ils se disent plus en surcharge de travail, ce que la littérature existante décrit comme étant l'une des causes de stress les plus importantes $[10,23]$.

Ils souffrent également de l'intensification du travail et de l'accélération des cadences puisqu'ils décrivent un niveau de rentabilité difficile à atteindre, compliqué par l'existence de délais à respecter. Une intensité du travail trop forte ne permet pas le compromis indispensable entre les exigences de la tâche et la préservation de la santé [13].

Ils sont également plus nombreux à exprimer que leur tâche est mal définie, ce qui peut rendre difficile l'accès au sens du travail. Cela est aggravé par les injonctions contradictoires qu'ils rencontrent plus fréquemment aussi. Ces éléments sont des facteurs bien connus de souffrance mentale au travail [10].

Ils se disent souvent dérangés dans le travail ce qui est très couteux en énergie du fait des dissonances cognitives que cela provoque [24]. Ces conditions de travail sont accompagnées d'un manque de moyens humains.

Ils évoquent aussi davantage une difficulté à réaliser $d u$ " beau travail ", c'est-à-dire un travail qu'ils estiment être de qualité, exploitant leurs savoirs faire et répondant à leur valeurs techniques et déontologiques de métier. Cette qualité non atteinte peut venir perturber leurs éthiques professionnelles. La réalisation d'un travail que l'on juge de qualité selon ses valeurs est fondamentale dans la construction de la santé psychique. La littérature montre l'impact psychologique chez les salariés présentant une conscience professionnelle heurtée [7].

Ils subissent également plus fréquemment des mutations. Il a été décrit que les personnes ayant réalisé un suicide ou une tentative de suicide liée au travail avaient évoqué à leur médecin du travail des difficultés d'adaptation à un nouvel environnement professionnel (tâches, process, rythme, mutations, déclassements,...) entraînant une perte de repères que peuvent être les collègues, les lieux d'exercices et habitudes de travail [25]. Les changements brutaux d'organisation du 
travail peuvent favoriser chez des personnes "sensibles" des troubles de l'adaptation. Ces personnes dites "sensibles" sont en quelque sorte des sentinelles, devant alerter sur l'éventuelle existence d'une souffrance collective.

La sous-charge de travail est également plus souvent rencontrée chez les suicidants établissant un lien entre leur TS et leur travail mais le faible effectif obtenu pour cette variable ne permet pas de conclusion.

Les suicidants évoquant un lien entre leur TS et leur travail sont davantage concernés par les situations de job strain et active au travail comme décrit par le modèle de Theorell et Karasek. Ces situations au travail sont connues pour être liées au stress professionnel [20]. Les niveaux de latitude décisionnelle et de charge mentale semblent jouer un rôle dans les processus suicidaires liés au travail. Mais l'absence de collectif de métier prend une part probablement déterminante dans la fragilisation des travailleurs qui se retrouvent isolés et seuls pour faire face aux difficultés rencontrées dans le réel du travail. Ainsi, on constate que les suicidants appartenant au sous-groupe "TS liées au travail " sont davantage en situation d'iso strain (situation tendue au travail aggravée par un faible soutien social au travail).

\section{Le vécu dans l'entreprise comme élément essentiel de décompensation psychique}

Lorsque le travail est un motif évoqué de la TS, on retrouve de façon plus fréquente la présence d'un climat social dégradé dans l'entreprise. Des tensions avec la hiérarchie, des menaces de licenciement, des tensions avec les collègues, voir même des menaces ou humiliations sont alors mentionnées. La littérature montre que ces comportements altèrent la santé psychique des travailleurs puisqu'ils sont liés à l'apparition d'une symptomatologie anxio-dépressive. Mais l'impact de telles relations de travail dépasse le cadre individuel du salarié qui en est victime puisqu'elles peuvent également avoir des répercussions sur l'environnement psychosocial collectif [21]. Les collectifs de travail, même ceux qui fonctionnent dans un mode défensif, peuvent ne pas résister face à un ou plusieurs éléments d'organisations du travail tel que ceux cités cidessus et/ou face à un climat social dégradé. En dernier ressort, les stratégies défensives individuelles peuvent ne plus être suffisantes. Le travail peut devenir inapte à accueillir l'individu, le fragiliser, et l'amener à une décompensation psychique potentiellement sévère, voire au suicide [17].

L'étude a mis en évidence que des discriminations sur le handicap étaient plus fréquentes parmi les TS liées au travail, mais il faut être prudent sur cette variable du fait du faible effectif concerné. De même, le stress intense ressenti au travail est plus fréquent parmi les suicidants évoquant le travail comme facteur explicatif de la TS. Le stress lié au travail est plus difficilement supporté par les sujets ayant un trouble de personnalité ou tout du moins une vulnérabilité au stress. Chez ces derniers, un climat de travail dégradé est plus délétère et pourvoyeur de décompensation psychique. Encore une fois il peut s'agir du reflet d'une souffrance collective. En effet, une situation stressante trop intense ou qui dure excessivement dans le temps peut entraîner une décompensation psychopathologique des salariés, notamment par l'épuisement psychique que cette situation peut engendrer.

\section{La reconnaissance de l'investissement au travail}

La reconnaissance au travail tient une place fondamentale par le rôle qu'elle joue dans la conquête de l'identité dans le champ social [14]. Elle est la forme spécifique de la rétribution moralesubjective donnée au salarié en retour de l'engagement de sa subjectivité et de son ingéniosité [26]. Elle peut être d'ordre matériel (salaire, promotions...) ou symbolique (respect des supérieurs plus encore que celui des collègues, reconnaissance de l'effort fourni et de la qualité du travail réalisé. . .). Lorsque le travail réalisé est reconnu par les pairs comme étant du beau travail et par la hiérarchie comme étant un travail utile, les difficultés rencontrées dans le réel du travail se transforment en réussite ce qui permet d'accéder au plaisir au travail, voire à la sublimation [27]. Les suicidants établissant un lien entre leur travail et leur passage à l'acte sont plus nombreux à estimer que la reconnaissance professionnelle est insuffisante à la vue de tous leurs efforts. En effet, on retrouve plus fréquemment chez eux le sentiment d'être traité injustement au travail, de ne pas recevoir le respect mérité de la part des supérieurs et de ne pas bénéficier de promotions ou perspectives d'avancée de carrière suffisantes.

\section{Conclusion}

Une interprétation prudente des résultats s'impose à la vue du faible effectif de l'échantillon.

Cependant, à travers une description des conditions de travail de suicidants hospitalisés, cette étude met en lumière l'importance de l'analyse de l'organisation du travail. En effet, cette dernière peut avoir un impact majeur au plan individuel. Les suicidants évoquant un lien entre leur TS et leur travail rapportent plus fréquemment la présence d'éléments d'organisations pathogènes du travail, de climat social dégradé, ainsi que d'une mauvaise reconnaissance des efforts effectués au travail. Cela permet de mieux comprendre les liens existants entre les TS et les conditions de travail. Ces éléments doivent être recherchés lors de l'évaluation du risque psychosocial dans l'entreprise. Leurs corrections pourraient améliorer la prévention du risque suicidaire lié au travail.

\section{Références}

[1] Baudelot C, Establet, Roger. Suicide l'envers de notre monde. Paris: Éditions du Seuil; 2006.

[2] Moreira P, Prolongeau H. Travailler à en mourir. Flammarion; 2009, 243. 
[3] Margraff A, Graser M, Manaouil C. Prise en charge du suicide au titre de la réglementation sur les accidents du travail. Archives des Maladies professionnelles et de l'Environnement 2006; 3(67):513-20.

[4] Guignon N, Niedhammer I, Sandret N. Les facteurs psychosociaux au travail. Une évaluation par le questionnaire de Karasek dans l'enquête Sumer. DARES 2008 2003;22(1).

[5] Karasek RA. Job demands, job decision latitude and mental strain : implications for job redesign. Admin Sci Quart 1979;24:285-307.

[6] Cohidon C, Murcia M, et al. Samotrace-Volet "Épidémiologie en entreprise". Résutats intermédiaires à un an (3000 questionnaires). Régions Centre, Pays de la Loire et Poitou-Charentes et le Comité de pilotage de Samotrace. Collection "Santé et travail". Mai 2007.

[7] Cohidon C. Samotrace point d'étape. Disponible à l'adresse URL : http://www.smt-dauphine-savoie.fr/2008-06-20/SocMed Wo608.pdf

[8] Niedhammer I, Siegrist J. Facteurs psychosociaux au travail et maladies cardio-vasculaires: l'apport du modèle Déséquilibre Efforts/Récompenses. Rev Epidem Et Santé Publ 1998;46: 398-410.

[9] Siegrist J, Starke D, Chandola T, et al. The measurement of effort-reward imbalance at work: European comparisons. Soc Sci Med 2004;58:1483-99.

[10] Chini B. Les facteurs de stress professionnel. Enquête auprès des salariés des services interentreprises. Arch Mal Prof 2003;64(5):297-309.

[11] Dejours C, Bègue F. Suicide et travail: que faire?, $1^{\mathrm{e}}$ éd, Paris: PUF; 2009. p. 129.

[12] Dejours C. Le facteur humain du travail, 4 éd., Paris: PUF; 2007. p. 127.

[13] Gollac M, Volkoff S. Les conditions de travail, $2^{\mathrm{e}}$ Ed., Paris: Repères; 2007. p. 121.

[14] Molinier P. Les enjeux psychiques du travail, $2^{\mathrm{e}}$ Ed., Paris: Payot et Rivages; 2006. p. 335.
[15] Bonde J. P E. Psychosocial factors at work and risk of depression: asystematic review of the epidemiological evidence. Occup Environ Med 2008;65:438-45.

[16] François S, Garre JB, Guiho-Bailly MP, et al. Étude exploratoire des caractéristiques professionnelles d'un échantillon de suicidants hospitalisés. Santé publique 2011;2(23):101-12.

[17] Dejours C. Souffrance en France. Éditions du Seuil; 1998.

[18] Suicides et tentatives de suicide en France. Études et résultats. Direction de la Recherche, des Études, de l'Évaluation et des Statistiques 2006; 488. Disponible à l'adresse URL: http:// www.sante.gouv.fr/drees/etude-resultat/er488/er488.pdf

[19] Touranchet A, Derriennic F, Volkoff S. Enquête ESTEV: un instrument d'étude des relations entre âge, santé et travail. Arch Mal Prof 1992;53(2):79-89.

[20] Guignon, N., Niedhammer, I., Sandret, N. Les facteurs psychosociaux au travail. Une évaluation par le questionnaire de Karasek dans l'enquête Sumer 2003; DARES 2008 No 22. 1.

[21] Parent-Thirion, A., Fernández Macías, E., Hurley, J., Vermeylen, G. Quatrième enquête européenne sur les conditions de travail. Fondation européenne pour l'amélioration des conditions de vie et de travail 2007; Luxembourg, Office des publications officielles des Communautés européennes.

[22] Indicateurs provisoires de facteurs de risques psychosociaux au travail. Collège d'expertise sur le suivi statistique des risques psychosociaux au travail. DARES DREES. 2009.

[23] Amagasa T, Nakayama T, Takahashi Y. Karojisatsu in Japan: characteristics of 22 cases of work-related suicide. J Occup Health 2005;47:157-64.

[24] Dejours C. Travail usure mentale, $3^{\mathrm{e}}$ éd., Paris: Bayard; 2000, 298.

[25] Gournay M, Lanièce F, Kryvenac I. Étude des suicides liés au travail en Basse-Normandie. Travailler 2004;12:91-8.

[26] Dejours C. L'évaluation du travail à l'épreuve du réel critique des fondements de l'évaluation. Paris: INRA; 2003, 82.

[27] Dessors Dominique. De l'ergonomie à la psychodynamique du travail. Éditions ERES. 2009 